\title{
Role of habit in treatment adherence among adults with cystic fibrosis
}

\author{
Zhe Hui Hoo, ${ }^{1,2}$ Benjamin Gardner, ${ }^{3}$ Madelynne A Arden, ${ }^{4}$ Simon Waterhouse, ${ }^{5}$ \\ Stephen J Walters, ${ }^{1}$ Michael J Campbell, ${ }^{1}$ Daniel Hind ${ }^{5}{ }^{5}$ Chin Maguire, ${ }^{5}$ Jane Dewar, ${ }^{6}$ \\ Martin J Wildman ${ }^{1,2}$
}

- Additional material is published online only. To view please visit the journal online (http://dx.doi.org/10.1136/ thoraxjnl-2017-211453).

${ }^{1}$ School of Health and Related Research (ScHARR), University of Sheffield, Sheffield, UK ${ }^{2}$ Sheffield Adult CF Centre, Northern General Hospital, Sheffield, UK

${ }^{3}$ Department of Psychology, Institute of Psychiatry, Psychology and Neuroscience (IoPPN), King's College London, London, UK

${ }^{4}$ Centre for Behavioural Science \& Applied Psychology (CeBSAP), Sheffield Hallam University,

Sheffield, UK

${ }^{5}$ Sheffield Clinical Trials Research Unit (CTRU), University of Sheffield, Sheffield, UK ${ }^{6}$ Department of Respiratory Medicine, Wolfson Cystic Fibrosis Centre, Nottingham University Hospitals NHS Trust, Nottingham, UK

Correspondence to Dr Zhe Hui Hoo, School of Health and Related Research (ScHARR), University of Sheffield, Sheffield S1 4DP, UK; z.hoo@sheffield.ac.uk

Received 20 December 2017 Revised 15 May 2018 Accepted 21 May 2018 Published Online First

9 June 2018

Check for updates

To cite: Hoo ZH, Gardner B, Arden MA, et al. Thorax 2019:74:197-199.

\section{ABSTRACT}

Among adults with cystic fibrosis (CF), medication adherence is low and reasons for low adherence are poorly understood. Our previous exploratory study showed that stronger 'habit' (ie, automatically experiencing an urge to use a nebuliser) was associated with higher nebuliser adherence. We performed a secondary analysis of pilot trial data $(n=61)$ to replicate the earlier study and determine whether habit-adherence association exists in other cohorts of adults with CF. In this study, high adherers also reported stronger habit compared with low adherers. Habit may be a promising target for self-management interventions. Trial registration number ACtiF pilot,

\section{ISRCTN13076797.}

\section{INTRODUCTION}

Adherence to preventative inhaled treatments is associated with better health outcomes in cystic fibrosis (CF), but adherence levels are generally low. ${ }^{1}$ Effective adherence interventions are lacking, ${ }^{2}$ and reasons for low adherence are poorly understood. Studies of adherence determinants in CF have tended to focus on treatment burden and conscious motivational factors such as treatment beliefs. ${ }^{2}$ However, behavioural theories such as the COM-B model (capability, opportunity, motivation and behaviour) propose a wider range of adherence predictors, including automatic processes such as habit. ${ }^{3}$ Indeed, habit may better predict treatment adherence compared with conscious motivational factors. ${ }^{4}$ Although commonly used to refer to frequent repetitive actions, within psychology the term 'habit' describes a non-conscious process by which situational cues (eg, time of day) automatically prompt an impulse to perform an action (ie, using nebuliser). ${ }^{5}$

Because they are automatically cued, habitual behaviours require little conscious cognitive effort to enact. ${ }^{5}$ Habits form through consistent repetition of a specific action in a specific context, which gradually shifts the cognitive control over initiation of action from reflective to automatic processes, such that encountering the context becomes sufficient to elicit the associated action. ${ }^{5}$ This reduces dependence on conscious attention or deliberative processes to initiate action. Habitual behaviours are thus thought to persist even if attention and conscious motivation wane. ${ }^{5}$

Our earlier exploratory mixed-methods study in Sheffield showed that high adherers had stronger habit than did low adherers. ${ }^{6}$ High adherers also described automatically 'remembering' to use their nebulisers; for example, one participant experienced the urge to use his nebuliser after walking his dog every morning. ${ }^{6}$ To determine whether the habit-adherence association exists in other cohorts of adults with CF, we performed a secondary analysis using data prospectively collected during a pilot randomised controlled trial (RCT) (ACtiF pilot, ISRCTN13076797). This two-centre pilot was primarily designed to explore the use of CFHealthHub, a software platform which delivers a complex intervention to support habit formation and self-management with inhaled therapies among adults with CF.

\section{METHODS}

Participants were recruited from the Wolfson Cystic Fibrosis Centre $(n=29)$ and the Wessex Adult CF Centre $(n=32)$ from June to September 2016. Nebuliser habit strength was measured at the point of recruitment using the Self-Report Behavioural Automaticity Index (SRBAI). ${ }^{7}$ The SRBAI consists of four statements (eg, 'deciding to use my nebuliser is something I do automatically') with which participants rate agreement on a 1-5 scale, where 5 represents strongest habit. Item scores were then summed to create a scale ranging from 4 (weakest habit) to 20 (strongest habit). Adherence data were downloaded from chipped nebulisers (eTrack) in the 3-month period following the point of recruitment, and calculated as 'normative adherence', ${ }^{8}$ a measure that takes into account a person's characteristics when defining the minimum required treatment regimen (see online supplementary appendix). Participants were divided into three adherence categories ( $<50 \%$ for low adherence, $50 \%-79.9 \%$ for moderate adherence, $\geq 80 \%$ for high adherence) to mirror the analysis of our earlier Sheffield study. ${ }^{6}$ These adherence categories were used in various other CF-related studies and were chosen based on the relationship with health outcomes. ${ }^{19}$

Outcomes data $\left(\mathrm{eg}, \mathrm{FEV}_{1}\right)$ and other psychological measures were also collected as part of the pilot trial (eg, beliefs about medicine necessity and concerns, which were combined in the Beliefs about Medicines Questionnaire - specific nebuliser adherence, ${ }^{10}$ a validated self-report tool that was customised for the pilot trial to identify perceived necessities and concerns for nebuliser treatment). Further details of the study variables are provided in the online supplementary appendix. Non-parametric statistical analysis methods were used, as 
Table 1 Clinical characteristics and psychological factors among the three groups of adults with CF

\begin{tabular}{|c|c|c|c|c|}
\hline & $\begin{array}{l}\text { Low adherence } \\
<50 \% \\
n=46\end{array}$ & $\begin{array}{l}\text { Moderate adherence } \\
50 \%-79.9 \% \\
n=8\end{array}$ & $\begin{array}{l}\text { High adherence } \\
\geq 80 \% \\
\mathrm{n}=7\end{array}$ & $P$ values* \\
\hline$\%$ Normative adherence, median (IQR) & $15.8(4.5-35.4)$ & $65.5(56.0-75.8)$ & $91.5(80.2-96.7)$ & \\
\hline \multicolumn{5}{|l|}{ Clinical characteristics } \\
\hline Age in years, median (IQR) & $27.4(21.7-37.1)$ & $23.7(18.4-32.0)$ & $26.1(21.2-37.5)$ & 0.616 \\
\hline Female, $\mathrm{n}(\%)$ & $22(47.8)$ & $5(62.5)$ & $1(14.3)$ & 0.164 \\
\hline Baseline $\% \mathrm{FEV}_{1}$, median (IQR) & $49.5(43.1-66.4)$ & $83.5(43.1-66.4)$ & $79.7(52.7-89.1)$ & 0.068 \\
\hline Prior-year IV days, median (IQR) & $28(13-47)$ & $15(4-53)$ & $0(0-10)$ & 0.012 \\
\hline IV days during trial, median (IQR) & $14(0-23)$ & $15(0-30)$ & $0(0-0)$ & 0.160 \\
\hline Quality of life (CFQ-R), median (IQR) & $50(28-78)$ & $64(34-88)$ & $67(44-78)$ & 0.306 \\
\hline \multicolumn{5}{|l|}{ Psychological factors } \\
\hline Anxiety (GAD) score, median (IQR) & $4(1-7)$ & $1(1-4)$ & $0(0-5)$ & 0.186 \\
\hline Depression (PHQ-8) score, median (IQR) & $6(3-11)$ & $6(3-13)$ & $4(2-4)$ & 0.389 \\
\hline Intention (COM-B) score, median (IQR) & $7(5-7)$ & $7(7-7)$ & $7(5-7)$ & 0.256 \\
\hline Necessity (BMQ) score, median (IQR) & $3.1(2.7-3.8)$ & $3.3(2.8-3.9)$ & $3.4(2.7-4.6)$ & 0.496 \\
\hline Concerns (BMQ) score, $†$ median (IQR) & $2.3(1.9-2.6)$ & $1.9(1.5-2.3)$ & $1.6(1.3-1.7)$ & 0.030 \\
\hline Habit (SRBAI) scores, $\uparrow$ median (IQR) & $9.0(4.8-12.0)$ & $14.5(11.3-18.3)$ & $18.0(14.0-20.0)$ & $<0.001$ \\
\hline
\end{tabular}

The values in bold indicates results that are statistically significant.

*All $\mathrm{p}$ values were calculated using Kruskal-Wallis $\mathrm{H}$ test, except the $\mathrm{p}$ value for gender which was calculated using Fisher's exact test.

tSince concerns and habit scores were associated with adherence, both scores were included as covariates in a multiple ordinal regression model with adherence category as the dependent variable. In this model, only habit strength was independently associated with adherence:

Pseudo- $r^{2}$ of model $=0.408$ (Nagelkerke); model $\chi^{2}(2)=22.9, p<0.001$.

Adjusted OR for concerns score $=0.344(95 \% \mathrm{Cl} 0.103$ to $1.152, \mathrm{p}=0.083)$.

Adjusted OR for habit score $=1.313(95 \% \mathrm{Cl} 1.123$ to $1.536, \mathrm{p}=0.001)$.

BMQ, Beliefs about Medicines Questionnaire; CF, cystic fibrosis; CFQ-R, Cystic Fibrosis Questionnaire-Revised respiratory domain; COM-B, capability, opportunity, motivation and behaviour; GAD, General Anxiety Disorder 7-item anxiety scale; PHQ-8, Patient Health Questionnaire depression scal; SRBAI, Self-Report Behavioural Automaticity Index.

well as multiple ordinal regression with adherence category as the dependent variable.

\section{RESULTS}

High adherers $(n=7)$ had lower prior-year intravenous use and tended to have higher $\% \mathrm{FEV}_{1}$ at baseline. High adherers reported stronger habit (median 18.0, IQR 14.0-20.0) than did low adherers $(n=46$; median 9.0, IQR 4.8-12.0) (see table 1). Conversely, high adherers reported lower concerns (median 1.6, IQR 1.3-1.7) than did low adherers (median 2.3, IQR 1.9-2.6). In a multiple ordinal regression model with both habit and concerns scores, only habit was associated with adherence. The adjusted OR indicated that a 1-unit increase in habit score was associated with a $31 \%$ increase in the odds of being in the nexthigher adherence category (95\% CI $12 \%$ to $54 \%$ ) (see footnote of table 1).

This two-centre pilot was not powered to determine the association between adherence and health outcomes. The absence of association between adherence and outcomes should not, therefore, be interpreted as evidence of absence. ${ }^{11}$

\section{DISCUSSION}

We had previously found the association between stronger habit and higher nebuliser adherence in Sheffield (for our earlier study, adherence was assessed retrospectively in the 1 -year prerecruitment period). In this study, our earlier finding has been replicated in a larger sample of adults with CF from two other centres (note that adherence was measured prospectively over a 3-month postrecruitment period in this study). This suggests that habit-that is, automatically experiencing an urge to use the nebuliser in certain settings, due to learnt associations between nebuliser use and cues within those settings-may be consistently associated with treatment adherence among adults with CF.

While habit is not the only factor associated with nebuliser adherence, appreciating the importance of both non-conscious motivation (eg, habit) and conscious motivation (eg, treatment beliefs) could lead to more effective adherence interventions. $^{35}$ The relationship between habit and adherence over time is complex and dynamic; initial repetitions of a behaviour (eg, using a nebuliser) will strengthen habit, which subsequently acquires the potential to direct subsequent performance. ${ }^{5}$ While studies of the formation of adherence habits are needed to reveal the causal direction or directions of the habit-adherence relationship we observed, our results are nonetheless consistent with the suggestion that, once formed, habit may determine subsequent adherence. ${ }^{56}$ Habit-formation advice, that is, encouraging the use of treatments in specific and unchanging contexts, so that associations may develop between those contexts and treatment adherence, is simple to deliver ${ }^{5}$ and could be part of a comprehensive package of interventions to support adherence. Habit strength might also be usefully assessed as an intervention outcome.

It is difficult to establish the causal direction of observed relationships in this study and the small sample size is a limitation (see online supplementary appendix for more detailed discussion regarding the limitation of the study). Nonetheless, we have replicated the Sheffield findings in an independent cohort, which suggests that the habit-adherence association is not just a peculiarity specific to a single adult CF centre. Two further studies are now under way to explore habit 
formation in more detail using CFHealthHub in a 19-centre RCT (ISRCTN55504164) and a three-centre improvement collaborative (ISRCTN14464661). We anticipate these studies will extend our understanding of the role of habit in sustained behaviour change among adults with CF.

Contributors ZHH, BG, MJW: concept and design. ZHH, SW, CM: data acquisition. ZHH: drafting the manuscript. All authors were involved in the analysis and interpretation of data, and critically revised the manuscript and approved the final draft.

Funding This report presents independent research funded by the NIHR under its Grants for Applied Research Programme (Grant Reference Number RPPG-1212-20015) and a Doctoral Research Fellowship (ZHH, Award Identifier DRF2014-07-092).

Disclaimer The views and opinions expressed in this publication are those of the authors and do not necessarily reflect those of the NHS, the NIHR, Medical Research Council (MRC), Central Commissioning Facility (CCF), NIHR Evaluation, Trials and Studies Coordinating Centre (NETSCC), the Programme Grants for Applied Research Programme, or the Department of Health.

Competing interests None declared.

Patient consent Obtained.

Ethics approval The Sheffield study was approved by the London - Westminster Research Ethics Committee (15/LO/0328). The ACtiF pilot study was approved by the London - Brent Research Ethics Committee (16/LO/0356).

Provenance and peer review Not commissioned; externally peer reviewed.

Data sharing statement Data for the Sheffield study are archived by the Sheffield Teaching Hospitals NHS Foundation Trust, and are available upon request (ZHH, clinical research fellow, z.hoo@sheffield.ac.uk) for researchers who meet the criteria for robust prespecified data analysis plan and for access to confidential data. Data for the two-centre pilot trial are archived by the University of Sheffield, and are available upon request (CM, trial manager, c.maguire@sheffield.ac.uk) for researchers who meet the criteria for robust prespecified data analysis plan and for access to confidential data. To access either data set, a formal request will need to be submitted and considered on a case-by-case basis.

(c) Article author(s) (or their employer(s) unless otherwise stated in the text of the article) 2019. All rights reserved. No commercial use is permitted unless otherwise expressly granted.

\section{REFERENCES}

1 Quittner AL, Zhang J, Marynchenko M, et al. Pulmonary medication adherence and health-care use in cystic fibrosis. Chest 2014;146:142-51.

2 Narayanan S, Mainz JG, Gala S, et al. Adherence to therapies in cystic fibrosis: a targeted literature review. Expert Rev Respir Med 2017;11:129-45.

3 Michie S, van Stralen MM, West R. The behaviour change wheel: a new method for characterising and designing behaviour change interventions. Implement $\mathrm{SC}$ 2011:6:42.

4 Phillips LA, Cohen J, Burns E, et al. Self-management of chronic illness: the role of 'habit' versus reflective factors in exercise and medication adherence. J Behav Med 2016;39:1076-91.

5 Gardner B. A review and analysis of the use of 'habit' in understanding, predicting and influencing health-related behaviour. Health Psychol Rev 2015;9:277-95.

$6 \mathrm{Hoo} \mathrm{ZH}$, Boote J, Wildman MJ, et al. Determinants of objective adherence to nebulised medications among adults with cystic fibrosis: an exploratory mixed methods study comparing low and high adherers. Health Psychol Behav Med 2017;5:299-316.

7 Gardner B, Abraham C, Lally P, et al. Towards parsimony in habit measurement: testing the convergent and predictive validity of an automaticity subscale of the Self-Report Habit Index. Int J Behav Nutr Phys Act 2012;9:102.

$8 \mathrm{Hoo}$ ZH, Curley R, Campbell MJ, et al. Accurate reporting of adherence to inhaled therapies in adults with cystic fibrosis: methods to calculate "normative adherence". Patient Prefer Adherence 2016;10:887-900.

9 Eakin MN, Bilderback A, Boyle MP, et al. Longitudinal association between medication adherence and lung health in people with cystic fibrosis. J Cyst Fibros 2011;10:258-64.

10 Horne R, Weinman J, Hankins M. The beliefs about medicines questionnaire: the development and evaluation of a new method for assessing the cognitive representation of medication. Psychol Health 1999;14:1-24.

11 Altman DG, Bland JM. Absence of evidence is not evidence of absence. BMJ 1995:311:485 